\title{
Accuracy of 3D acquisition mode for myocardial FDG PET studies using a BGO-based scanner
}

\author{
Arno P. van der Weerdt • Ronald Boellaard • \\ Frans C. Visser • Adriaan A. Lammertsma
}

Received: 18 September 2006 / Accepted: 26 December 2006 / Published online: 27 February 2007

(C) Springer-Verlag 2007

\begin{abstract}
Purpose The aim of the present study was to evaluate the quantitative and qualitative accuracy of 3D PET acquisitions for myocardial FDG studies.

Methods Phantom studies were performed with both a homogeneous and an inhomogeneous phantom. Activity profiles were generated along the phantoms using $2 \mathrm{D}$ and several $3 \mathrm{D}$ reconstructions, varying the $3 \mathrm{D}$ scaling value to adjust the scatter correction algorithm. Furthermore, ten patients underwent a dynamic myocardial FDG PET scan, using an interleaved protocol consisting of frames with alternating $2 \mathrm{D}$ and $3 \mathrm{D}$ acquisition. For each myocardial study, 13 volumes of interest were defined, representing 13 myocardial segments. First, the optimal scaling value for the scatter correction algorithm was determined using data from the phantom and four patient studies. This scaling value was then applied to all ten patients. $2 \mathrm{D}$ and $3 \mathrm{D}$ acquisitions were compared for both static (i.e. activity concentrations in the last 2D and 3D frames) and dynamic imaging (calculation of the metabolic rate of glucose).

Results For both phantom and patient studies, suboptimal results were obtained when the default scaling value for the scatter correction algorithm was used. After adjusting the
\end{abstract}

A. P. van der Weerdt $(\bowtie) \cdot$ F. C. Visser

Department of Cardiology, Institute for Cardiovascular

Research-VU, VU University Medical Centre,

De Boelelaan 1117,

1081 HV Amsterdam, The Netherlands

e-mail: a.vdweerdt@vumc.nl

R. Boellaard · A. A. Lammertsma

Department of Nuclear Medicine and PET Research,

Institute for Cardiovascular Research-VU,

VU University Medical Centre,

Amsterdam, The Netherlands scaling value, for all ten myocardial FDG studies, a very good correlation $\left(r^{2}=0.99\right)$ was obtained between $2 \mathrm{D}$ and $3 \mathrm{D}$ data. With the present protocol no significant differences were observed in qualitative interpretation.

Conclusion The 3D FDG acquisition mode is accurate and has clear advantages over the 2D mode for myocardial FDG studies. A prerequisite is, however, optimisation of the 3D scatter correction algorithm.

Keywords 3D $\cdot$ PET $\cdot{ }^{18} \mathrm{~F}-\mathrm{FDG} \cdot$ Scatter $\cdot$ Myocardial

\section{Introduction}

Conventional positron emission tomography (PET) scanners are equipped with interplane septa to limit the detection of scattered (especially in the axial direction) and random coincidences. A side-effect of these septa is that only a small fraction of all true coincidences in the axial field of view can be measured - in fact, only those within the range of a few planes.

Modern PET scanners have retractable or no interplane septa, thereby enabling a 3D scanning mode in which coincidences can be measured throughout the axial field of view. The system sensitivity of this 3D mode may be five to six times higher than that of the 2D mode ( $3 \%$ instead of $\sim 0.5 \%$ of all decaying nuclei) [1].

${ }^{18} \mathrm{~F}$-fluorodeoxyglucose (FDG) PET has been used extensively to visualise and quantify myocardial glucose metabolism [2-7], and it is an important tool for the detection of myocardial viability. Performing clinical studies in $3 \mathrm{D}$ rather than $2 \mathrm{D}$ acquisition mode may allow for the use of less activity and/or shortening of the study duration, without loss of image quality. The 3D scanning mode, however, is accompanied by an increase in the 
scattered events of up to $50 \%$ of the total measured coincidences $[8,9]$. Therefore, dedicated scatter correction methods are required [10]. Other problems affecting the accuracy of 3D PET relate to attenuation correction, detector normalisation and the increase in dead time and randoms [11].

Most clinical experience with 3D PET has been obtained with brain studies [12-14]. Data from cardiac studies are limited and conflicting [15-19]. Due to differences in scanner characteristics and imaging protocols, published results cannot be used without further validation.

The aim of the present study was to determine the accuracy of the 3D acquisition mode for both static and dynamic myocardial FDG studies as compared with 2D studies.

\section{Materials and methods}

\section{PET scanner}

All phantom and clinical studies were performed using an ECAT EXACT HR+ scanner (CTI/Siemens, Knoxville, $\mathrm{TN})$. This scanner is equipped with BGO crystals and acquires 63 planes of data over an axial field of view of $15 \mathrm{~cm}$. Scans can be acquired in both 2D and 3D mode. For attenuation correction, a transmission scan can be performed using three rotating ${ }^{68} \mathrm{Ge}$ line sources. The characteristics of this scanner have been described in detail elsewhere $[20,21]$. In the present study, all acquisitions and reconstructions were performed using ECAT software version 7.2.

\section{Reconstructions}

All emission scans were reconstructed using filtered back projection (FBP) with a Hanning filter at $5 \mathrm{~mm}$ full-width at half-maximum. In the case of 3D emission scans, reconstructions were performed using Fourier rebinning [22], followed by 2D FBP using the same settings and filters. For both $2 \mathrm{D}$ and $3 \mathrm{D}$ studies the resulting image resolution equalled $6.8 \mathrm{~mm}$ at the centre of the field of view (FOV). Normalisation, attenuation, scatter, dead time and decay corrections were included in all reconstructions.

\section{Scatter correction}

For the purpose of the present study, the standard 3D scatter algorithm provided by the manufacturer, which involves a simulation-based method [23], was modified to allow variation of the scaling (amplitude) of the scatter correction. Although other parameters, such as energy window settings or the number of simulations point, could also be varied, the scaling (amplitude) of the scatter correction is a major determinant of quantitatively accurate scatter correction, as will be discussed in detail later. The default scaling is set to 2.3 , which is an arbitrary value defined by the manufacturer. To evaluate the effects of the scaling of the scatter correction on the accuracy of $3 \mathrm{D}$ scans, several 3D emission scans were reconstructed with various scatter scaling values $(0,1,2.3,3.5,4,4.5,5$ and 6$)$ using both phantom and clinical studies. Changing the scaling value of the scatter correction algorithm affects the absolute values of measured activity in the whole data set and therefore requires an adjustment of the scanner calibration. The optimal calibration factor was obtained from the phantom studies. Subsequently, the optimal scaling value and calibration factor were used for reconstruction of all clinical 3D emission scans. For reconstruction of 2D emission scans, the default reconstruction software and (scatter) corrections were used $[20,21]$. The 2D scatter correction involves a stationary convolution subtraction method [24].

\section{Phantom studies}

A number of phantom studies were performed to evaluate the quantitative accuracy of $3 \mathrm{D}$ versus $2 \mathrm{D}$ acquisition modes and to optimise calibration of the scanner and (scaling of the) 3D scatter correction algorithm. The following phantoms were used: (a) a homogeneous 20-cm-long, 20-cm-diameter phantom filled with an FDG solution of $\sim 15 \mathrm{kBq} / \mathrm{ml}$ to evaluate the accuracy of the calibration of the scanner; (b) the same phantom, but now extended with an additional $20-\mathrm{cm}$-long phantom filled with the same FDG solution to simulate outside FOV activity; (c) the NEMA NU-2 1994 scatter phantom to verify the accuracy of the scatter correction algorithm. The latter phantom is a $20-\mathrm{cm}$-long, 20-cm-diameter phantom with a $5-\mathrm{cm}$-diameter water-filled insert positioned $5 \mathrm{~cm}$ from the axis of the phantom. The large background compartment was filled with an FDG solution of $\sim 15 \mathrm{kBq} / \mathrm{ml}$, while the insert contained no activity $(0 \mathrm{kBq} / \mathrm{ml})$. For all these phantoms, both $15-\mathrm{min} 2 \mathrm{D}$ and 15-min 3D emission scans were acquired. These scans showed approximately $2.9 \mathrm{E} 7$ and $1.4 \mathrm{E} 8$ total numbers of trues and random fractions of about 0.18 and 0.30 for $2 \mathrm{D}$ and 3D scans, respectively. After activity had decayed to background levels (after $12 \mathrm{~h}$ ) a 15-min transmission was performed for attenuation and scatter correction purposes. Data were reconstructed as described above using various scatter scaling values in the case of $3 \mathrm{D}$ reconstructions.

Analysis of phantom studies

The activity concentration in a 15 -cm-diameter region of interest (ROI), averaged over all image planes, in 
phantom (a) for both 2D and 3D scans was calculated to verify the calibration of the scanner for $2 \mathrm{D}$ and $3 \mathrm{D}$ scans. Furthermore, 2-cm-wide horizontal and vertical profiles through the centre of the phantom were derived. The effect of scaling the $3 \mathrm{D}$ scatter correction on the level and variation of the reconstructed activity concentration along the phantom $x$ - and $y$-axis, was investigated. Next, from phantom (b) the effect of outside FOV activity on interplane variation of average activity concentration was determined using a 15 -cm-diameter ROI. Finally, to determine the accuracy of the scatter correction algorithm, a 2.5-cm ROI was placed within the cold insert of the NEMA scatter phantom (phantom c) and several $5-\mathrm{cm}$ ROIs were placed in the warm background compartment. The accuracy of the scatter correction algorithm was evaluated using the cold spot recovery coefficient (CSRC), defined here as the ratio between the measured activity concentration in the $2.5-\mathrm{cm}$ cold insert ROI and the average activity concentration in the 5 - $\mathrm{cm}$ background ROIs. In theory, accurate scatter correction would result in a CSRC equal to 0.0 .

\section{Clinical studies}

Ten consecutive patients with ischaemic heart disease were included. All patients had impaired left ventricular function due to previous myocardial infarction, and were referred for assessment of myocardial viability. All patients underwent an FDG PET scan during a euglycaemic hyperinsulinaemic clamp, a procedure that has been described elsewhere [25].

The study was approved by the Medical Ethics Committee of the VU University Medical Centre and informed consent was obtained from each patient.

\section{Clinical scanning protocol}

After a short transmission scan for patient positioning, a 10-min transmission scan was performed to correct the subsequent emission scan for attenuation. The emission scan itself consisted of 35 frames with variable frame length $(6 \times 5,6 \times 10,3 \times 20,5 \times 30,5 \times 60,10 \times 300 \mathrm{~s})$ and a total scan duration of $60 \mathrm{~min}$. The start of this scan coincided with the intravenous injection of $\sim 185 \mathrm{MBq}$ of FDG. During the first $10 \mathrm{~min}$ after injection, data acquisition was performed in 3D mode. Thereafter alternating $2 \mathrm{D}$ and $3 \mathrm{D}$ frames were acquired, each frame lasting $5 \mathrm{~min}$.

The input function was obtained from continuous arterial blood sampling [25]. At set times (5, 10, 20, 30 and $50 \mathrm{~min}$ after injection), continuous withdrawal was interrupted for collection of manual samples. These samples were measured in a well counter, cross-calibrated against the PET scanner and used for on-line calibration of the blood sampler and for determination of plasma to whole blood ${ }^{18} \mathrm{~F}$ ratios. In addition, these samples were used for measurement of plasma glucose levels (hexokinase method, Hitachi 747, Boeringer Mannheim, Mannheim, Germany).

Analysis of clinical studies

In four patients the last 3D frame of the interleaved dynamic scan was reconstructed as described above using various scaling values of the $3 \mathrm{D}$ scatter correction algorithm. All 2D frames of the interleaved dynamic scan were reconstructed using the default settings of the reconstruction software as provided by the manufacturer. After reconstruction, dynamic images were analysed using standard Siemens/CTI reslicing and ROI software. Transaxial images of the left ventricle were reoriented according to the anatomical axis of the heart and subsequently displayed as short axis slices. The last 3D frame was used for this reslicing procedure. Myocardial tissue ROIs were manually defined on the short axis image and projected onto both the $2 \mathrm{D}$ and the $3 \mathrm{D}$ data set. At the basal and distal levels of the left ventricle, these ROIs divided each short axis slice into six regions. At the apex, one ROI was defined on each short axis slice. Additionally, ROIs were defined at the basal level in the left ventricular cavity. For each patient, corresponding ROIs from a variable number of slices were grouped to compose 14 volumes of interest (VOIs), representing 13 myocardial segments (six basal, six distal and one apical) and one left ventricular cavity. Using these VOIs, activity concentrations of the last 3D frame and the average activity concentration of the last two $2 \mathrm{D}$ frames, which preceded and followed the last 3D frame, were determined. The ratio between $2 \mathrm{D}$ and $3 \mathrm{D}$ data was calculated for all scaling values applied. The latter data were used in conjunction with the phantom data to determine optimal scaling of the 3D scatter correction algorithm and to correct the calibration of the scanner, as will be discussed later.

Next, all 3D frames of the interleaved dynamic scan were reconstructed using these (optimal) settings for 3D scatter correction and scanner calibration for all patients.

Activity concentrations of the last 3D frame and the average activity concentration of the last two $2 \mathrm{D}$ frames were now determined for all ten patients. The ratio between $2 \mathrm{D}$ and $3 \mathrm{D}$ data was plotted against the average 2D activity concentration. Subsequently, the metabolic rate of glucose (MRGlu) was calculated using Patlak graphical analysis [26] over the period from 10 to 70 min post injection and for $2 \mathrm{D}$ and $3 \mathrm{D}$ acquisitions separately.

Finally, the ratio of MRGlu obtained with 2D and 3D PET was calculated. 
Statistical analysis

All data are expressed as mean values \pm SD. Statistical analyses were performed using the two-tailed paired $t$ test and least squares regression analysis. The limits of agreement were assessed by means of the analysis described by Bland and Altman [27]. A $p$ value $<0.05$ was considered statistically significant.

\section{Results}

Phantom studies

Using profiles along a homogeneous phantom, the effect of varying scatter correction scaling on reconstructed activity concentration is demonstrated in Fig. 1a. The default scaling (2.3) results in considerable overestimation. Applying scaling value 4.5 results in a more constant course and it approaches the $2 \mathrm{D}$ profile. This effect is also seen in the presence of activity outside the FOV (Fig 1b).

The CSRC linearly decreases with the use of higher scaling values for scatter correction (Fig. 2). When higher scaling values are used, the CSRC almost equals 0 .

\section{Clinical studies}

Ten patients (seven male, three female) with a mean age of $65 \pm 11$ years were studied. The mean patient weight was $84 \pm 17 \mathrm{~kg}$ and ranged from 70 to $126 \mathrm{~kg}$. The mean body mass index (BMI) was $28.6 \pm 6.5 \mathrm{~kg} / \mathrm{m}^{2}$ and ranged from 22.1 to $45.2 \mathrm{~kg} / \mathrm{m}^{2}$.

Activity in the myocardial VOIs of the 2D studies was on average $23.6 \pm 12.5 \mathrm{kBq} / \mathrm{ml}$ and ranged from 6.1 to $63.3 \mathrm{kBq} / \mathrm{ml}$.

First, in line with the phantom studies, the effect of varying the scatter correction scaling was evaluated in four consecutive patients. Low scaling factors (e.g. the default setting) resulted in overestimation of FDG concentration (especially when activity was below $10 \mathrm{kBq} / \mathrm{ml}$ ) using 3D as compared with $2 \mathrm{D}$ acquisitions. A high scaling value resulted in a considerable underestimation of FDG concentration in regions with low activity. The optimal value for both low and high activity levels (i.e. with the lowest SD) appeared to be 4.5 (Fig. 3, Table 1). Mean activity was overestimated by $10 \%$ using this scaling value (Table 1 ) and this information was subsequently used to adjust the $3 \mathrm{D}$ calibration factor of the scanner.

The optimal scaling value was then applied to all ten studies. A total of 130 segments were analysed. Activity in the last $2 \mathrm{D}$ and $3 \mathrm{D}$ frames showed very good correlation over a wide range of VOI values $\left(y=1.01 \times-0.07, r^{2}=0.99\right.$; mean difference $=0.24 \mathrm{kBq} / \mathrm{ml}, 2 \mathrm{SD}=2.40 \mathrm{kBq} / \mathrm{ml}$ ) (Fig. 4). a

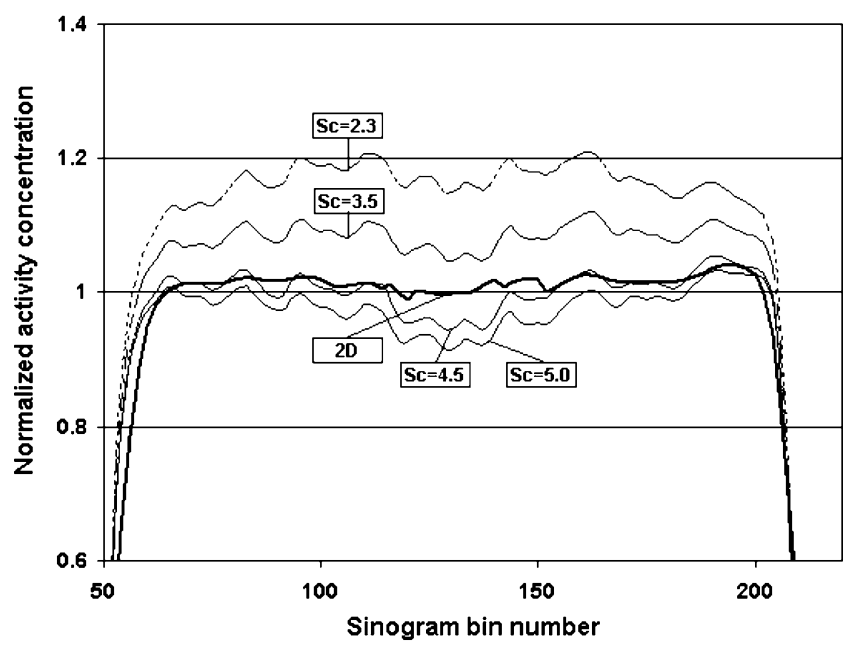

b

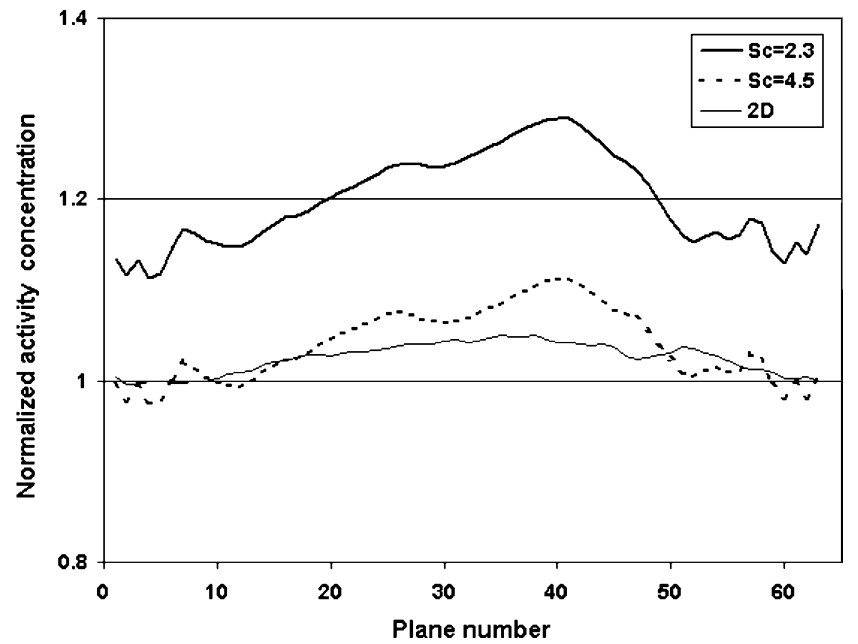

Fig. 1 a $2 \mathrm{D}$ profile of normalised activity concentration (ratio between reconstructed phantom activity concentration and that obtained from samples in a well counter) along a homogeneous phantom with corresponding $3 \mathrm{D}$ profiles with varying scaling values (Sc) applied to the scatter correction. b Effect of outside FOV activity on measured activity concentration per plane using $2 \mathrm{D}$ reconstruction and $3 \mathrm{D}$ with two different scaling values applied to the scatter correction

Almost identical results were obtained when the analysis was performed without the four patients who were used to determine the optimal scaling value $(y=1.04 \times-0.54$; $r^{2}=0.98$; mean difference $=0.41 \mathrm{kBq} / \mathrm{ml}, 2 \mathrm{SD}=2.78 \mathrm{kBq} / \mathrm{ml}$, $n=6)$.

Mean MRGlu was $269 \pm 119 \mu \mathrm{mol} / \mathrm{ml} / \mathrm{min}$ with $2 \mathrm{D}$ acquisition and $274 \pm 128 \mu \mathrm{mol} / \mathrm{ml} / \mathrm{min}$ with $3 \mathrm{D}$ acquisition $(p<0.001) .2 \mathrm{D}$ and 3D MRGlu values were closely correlated $\left(y=1.07 \times-0.01 ; r^{2}=0.99 ;\right.$ mean difference $=5 \mu \mathrm{mol} / \mathrm{ml} / \mathrm{min}$, $2 \mathrm{SD}=31 \mu \mathrm{mol} / \mathrm{ml} / \mathrm{min}$ ) (Fig. 5). Performing the analysis without the four patients who were used to determine the optimal scaling value did not change the results $(y=$ $1.09 \times-0.02 ; r^{2}=0.98 ;$ mean difference $=10 \mu \mathrm{mol} / \mathrm{ml} / \mathrm{min}$, $2 \mathrm{SD}=34 \mu \mathrm{mol} / \mathrm{ml} / \mathrm{min}, n=6$ ). 


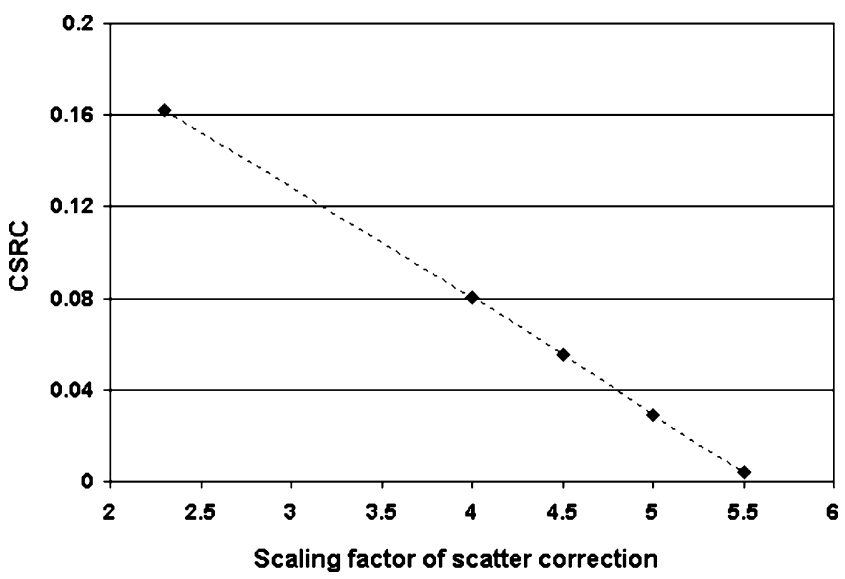

Fig. 2 Linear plot through the cold spot recovery coefficient (CSRC) for different $3 \mathrm{D}$ scatter scaling values. The CSRC for $2 \mathrm{D}$ acquisition was $0.025 \pm 0.05(1 \mathrm{SD})$

\section{Qualitative analysis}

With one exception, all images were of good quality with only small differences between $2 \mathrm{D}$ and $3 \mathrm{D}$ images, the latter being slightly smoother in general appearance (Fig. 6). As no difference in image resolution was observed, this is the result of differences in counting statistics. 2D and 3D images of one study were of inferior quality, probably owing to insulin resistance and adiposity of the patient.

\section{Discussion}

The purpose of the present study was to evaluate the quantitative and qualitative accuracy of 3D FDG PET in cardiac studies. As 3D acquisitions may introduce bias due to a significant increase in scattered events as compared with $2 \mathrm{D}$, accuracy of the scatter correction algorithm plays a prominent role in $3 \mathrm{D}$ image reconstruction.

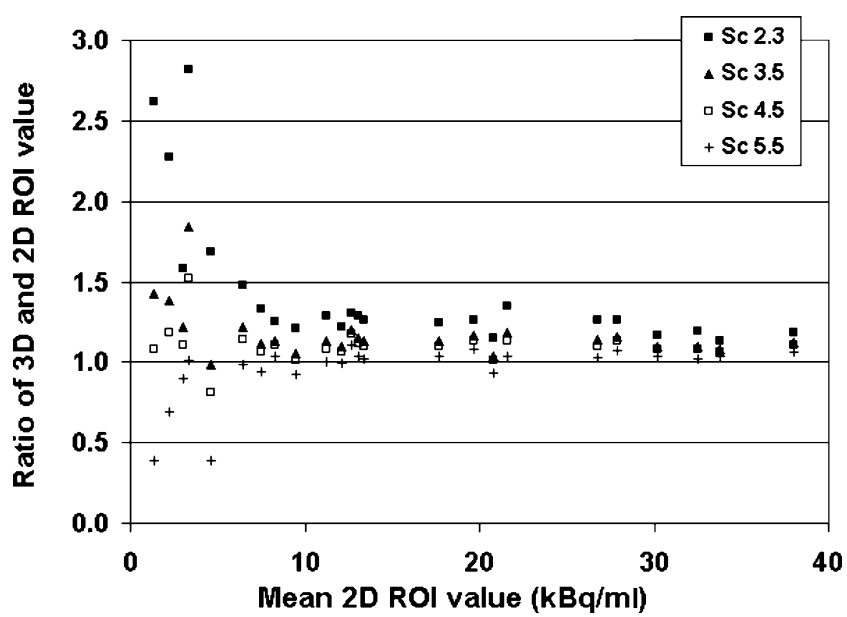

Fig. 3 Ratio of 3D and 2D FDG concentration using different scaling values $(S c)$ for scatter correction in $3 \mathrm{D}$ reconstructions, as a function of the $2 \mathrm{D}$ ROI value
Table 1 Mean ratio and SD of activity between 3D and 2D reconstructions using different scaling values for $3 \mathrm{D}$ scatter correction

\begin{tabular}{lllll}
\hline & Sc 2.3 & Sc 3.5 & Sc 4.5 & Sc 5.5 \\
\hline Mean & 1.45 & 1.18 & 1.10 & 0.95 \\
SD & 0.46 & 0.17 & 0.11 & 0.19 \\
\hline
\end{tabular}

Using phantom studies it was demonstrated that the scatter correction algorithm was suboptimal when the default settings (scaling value of 2.3) of the manufacturer were used. Adjusting the scaling value resulted in 3D data that more closely resembled data from 2D acquisitions. A scaling value of 4.5 was considered to be optimal as the absolute difference in measured activity with 2D was minimal. Moreover, the activity profile along the phantom showed fewer fluctuations, making it easier to determine the $3 \mathrm{D}$ calibration factor. These effects were also seen in the presence of activity outside the FOV, a situation that is normal for clinical myocardial FDG PET studies because of high FDG uptake in the liver. Furthermore, only with high
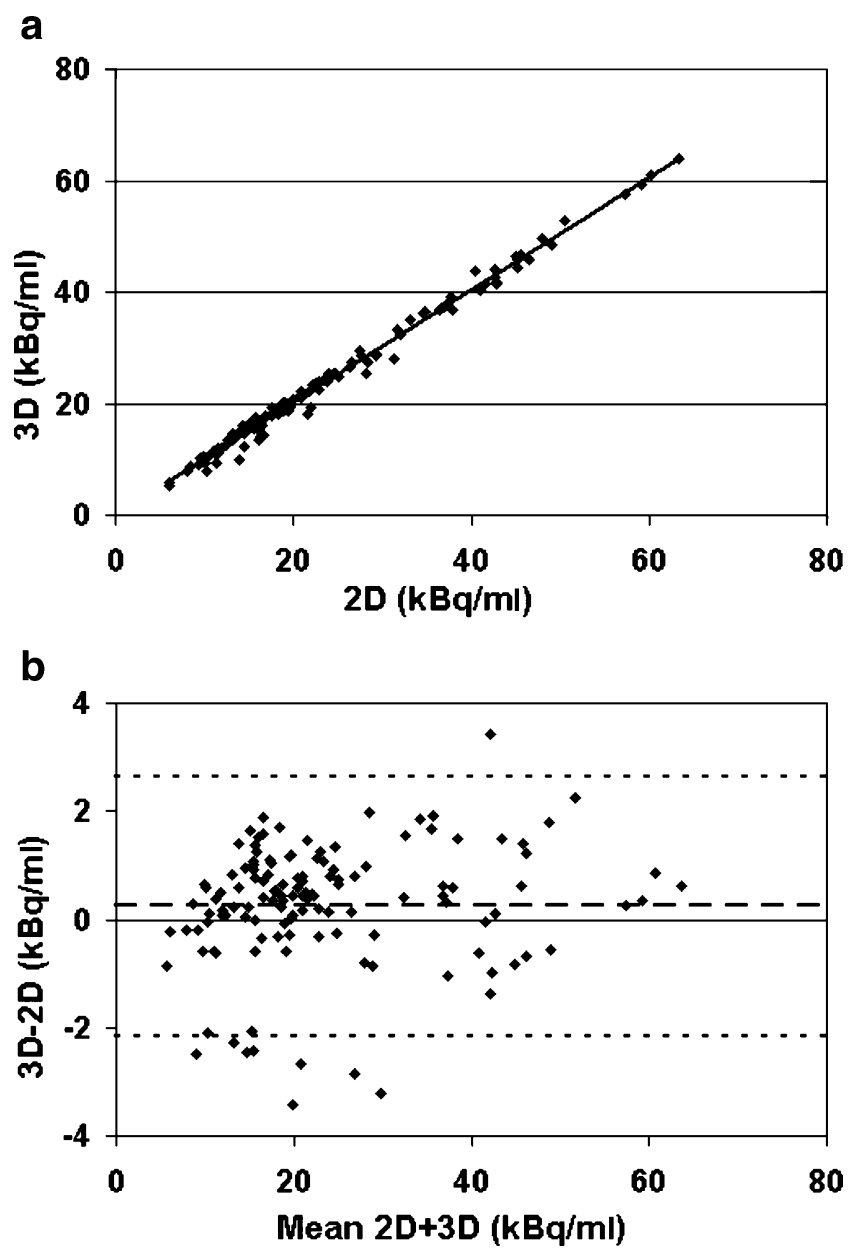

Fig. 4 a Scatterplot of the correlation between activity in the last 3D frame and the mean activity in the last two 2D frames. b Limits of agreement between 2D and 3D ROI values. Horizontal lines represent the mean difference and mean $\pm 2 \mathrm{SD}$ 


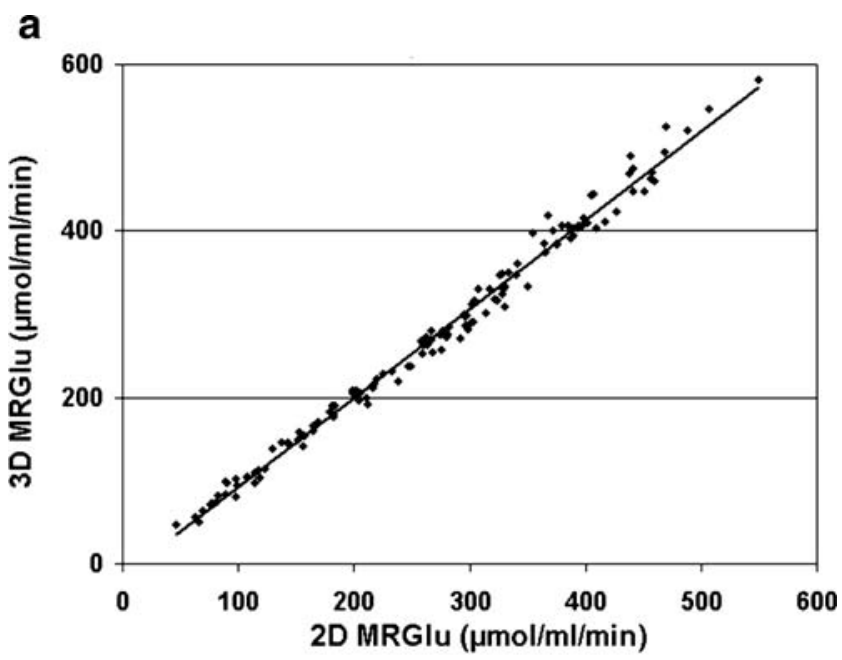

b

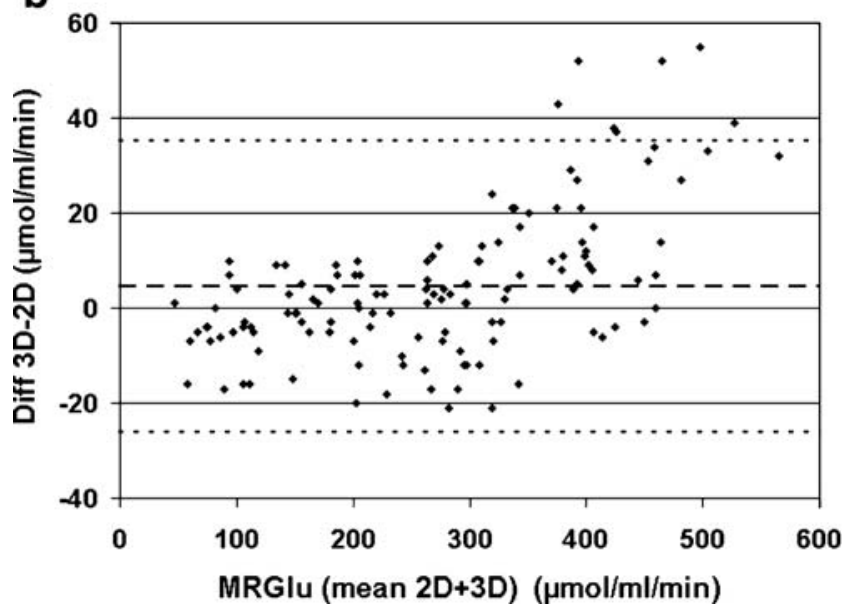

Fig. 5 a Scatterplot of the correlation between 2D and 3D MRGlu. b Limits of agreement between 2D and 3D MRGlu. Horizontal lines represent the mean difference and mean $\pm 2 \mathrm{SD}$

scaling values could a low CSRC be obtained for the scatter phantom.

To further explore these findings in a clinical setting, four consecutive myocardial FDG studies were used to reconstruct the same (static) images with varying scatter scaling values. In accordance with the results of the phantom studies, for the default scatter scaling factor, FDG concentration was overestimated compared with 2D data, especially in areas with low FDG concentrations $(<10 \mathrm{kBq} / \mathrm{ml})$. In contrast, high scaling values resulted in severe underestimation in areas with low FDG concentrations (Fig. 3).

The best results, over a wide range of FDG concentrations, were obtained with a scaling value of 4.5. This was also reflected in the lowest SD of all scaling values tested (Table 1). As 4.5 was considered to be the optimal scaling value, it was applied to all ten myocardial FDG PET studies.

Using this scatter scaling value, FDG concentrations of static $3 \mathrm{D}$ and $2 \mathrm{D}$ frames showed a good correlation. One

study was of inferior quality in both $2 \mathrm{D}$ and $3 \mathrm{D}$ acquisition [probably due to obesity (BMI $45.2 \mathrm{~kg} / \mathrm{m}^{2}$ ) and insulin resistance of the patient] and resulted in underestimation of FDG concentrations with 3D acquisition. Increased scatter in this heavy patient may have contributed to the differences between 2D and 3D results. The impaired image quality with increasing patient weight in 3D PET scanning has already been addressed [28-30]. However, no data were discarded with respect to the statistical analyses.

The same favourable results were obtained when a comparison was made between MRGlu values calculated for $2 \mathrm{D}$ and $3 \mathrm{D}$ data separately, although a small overestimation at high activity levels was observed with 3D acquisition. Probably this is still due to a suboptimal scatter scaling value and 3D calibration factor. A different behaviour of the 3D scatter correction algorithm with changing activity distribution during the dynamic scan could also contribute to this finding as this pattern was not observed when the activity levels of the last $2 \mathrm{D}$ and $3 \mathrm{D}$ frames of the dynamic scan were compared. It is likely that the small differences between the 2D and 3D data will have no clinical significance.

The good correlation of $2 \mathrm{D}$ and $3 \mathrm{D}$ data is in line with earlier reports comparing 2D and 3D FDG PET [18, $19,29]$. However, in a recent study inaccuracy of 3D imaging was demonstrated especially in regions with low FDG uptake, probably reflecting a suboptimal scatter correction [19].

In the present study, comparing a $10-$ min $2 \mathrm{D}$ emission image with a 5-min 3D emission image, no significant differences were observed in image quality and qualitative interpretation. This supports the use of 3D acquisition in clinical practice.

\section{Limitations}

The four patients from whom the optimal scaling value was derived were part of the final study population and this could be a confounding factor. However, excluding the four

2D

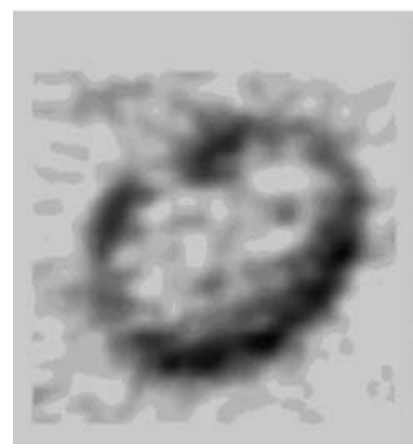

Fig. 6 Representative examples of transaxial 2D and 3D images 
patients from the analysis did not significantly affect the results.

This procedure contains both phantom and clinical studies. Clearly, more phantom studies could have been included. The clinical results indicate, however, that use of the phantoms and experiments as described in the NEMA NU2 1994 protocols is sufficient.

The present results are only applicable to the HR+ scanner with its specific reconstruction software (version 7.2.1) using a procedure aimed at optimising the accuracy of $3 \mathrm{D}$ acquisitions and reconstructions. It should be noted that a similar evaluation is required for every scanner with its own specific reconstruction software before the accuracy of $3 \mathrm{D}$ acquisitions can be guaranteed. This is also true for PET/CT scanners, even if the PET geometry is the same as that of a conventional PET scanner. A larger gantry and possibly less internal side-shielding of detectors (due to the presence of the CT) may affect the results.

The present results were obtained with a PET scanner equipped with bismuth germanate (BGO) scintillation crystals. At present both lutetium oxyorthosilicate (LSO)based PET and PET/CT scanners are commercially available. As the scintillation decay time of LSO crystals is shorter and thus a smaller coincidence time window can be applied, randoms and dead time will be reduced and scatter rejection may be improved by a narrower energy window. Therefore, we expect that it should be possible to achieve at least comparable results as compared with BGO-based PET scanners [31]. However, accuracy of the 3D acquisition mode will be influenced by the same factors as are described in the present study. Furthermore, a substantial number of PET/CT scanners are still equipped with BGO crystals [32].

A dose of $\sim 185 \mathrm{MBq}$ FDG was injected to perform the clinical studies. As a consequence, results obtained in this study cannot be extrapolated to high count rate studies (e.g. in the case of ${ }^{82} \mathrm{Rb}$ and ${ }^{15} \mathrm{O}$ studies) as these result in a significant increase in dead time and randoms. Due to these effects, high count rate studies should not be performed using 3D acquisitions with a BGO-based scanner [17].

\section{References}

1. Bailey DL. 3D acquisition and reconstruction in positron emission tomography. Ann Nucl Med 1992;6:123-30.

2. Marshall RC, Tillisch JH, Phelps ME, Huang SC, Carson R, Henze $\mathrm{E}$, et al. Identification and differentiation of resting myocardial ischemia and infarction in man with positron computed tomography, ${ }^{18} \mathrm{~F}$-labeled fluorodeoxyglucose and N-13 ammonia. Circulation 1983;67:766-78.

3. Tillisch J, Brunken R, Marshall R, Schwaiger M, Mandelkern M, Phelps $M$, et al. Reversibility of cardiac wall-motion abnormalities predicted by positron tomography. N Engl J Med 1986;314:884-8.
4. Tamaki N, Yonekura Y, Yamashita K, Saji H, Magata Y, Senda M, et al. Positron emission tomography using fluorine-18 deoxyglucose in evaluation of coronary artery bypass grafting. Am J Cardiol 1989;64:860-5.

5. Schwaiger M, Brunken R, Grover-McKay M, Krivokapich J, Child J, Tillisch JH, et al. Regional myocardial metabolism in patients with acute myocardial infarction assessed by positron emission tomography. J Am Coll Cardiol 1986;8:800-8.

6. Marwick TH, MacIntyre WJ, Lafont A, Nemec JJ, Salcedo EE. Metabolic responses of hibernating and infarcted myocardium to revascularization. A follow-up study of regional perfusion, function, and metabolism. Circulation 1992;85:1347-53.

7. Gropler RJ, Geltman EM, Sampathkumaran K, Perez JE, Schechtman KB, Conversano A, et al. Comparison of carbon11-acetate with fluorine-18-fluorodeoxyglucose for delineating viable myocardium by positron emission tomography. J Am Coll Cardiol 1993;22:1587-97.

8. Bailey DL, Jones T. A method for calibrating three-dimensional positron emission tomography without scatter correction. Eur J Nucl Med 1997;24:660-4.

9. Badawi RD, Marsden PK, Cronin BF, Sutcliffe JL, Maisey MN. Optimization of noise-equivalent count rates in 3D PET. Phys Med Biol 1996;41:1755-76.

10. Zaidi H. Scatter modelling and correction strategies in fully 3-D PET. Nucl Med Commun 2001;22:1181-4.

11. Badawi RD. 3D-Mode acquisition in clinical PET. Nucl Med Commun 1997; 18:801-4.

12. Silbersweig DA, Stern E, Frith CD, Cahill C, Schnorr L, Grootoonk S, et al. Detection of thirty-second cognitive activations in single subjects with positron emission tomography: a new low-dose $\mathrm{H}_{2}{ }^{15} \mathrm{O}$ regional cerebral blood flow threedimensional imaging technique. J Cereb Blood Flow Metab 1993;13:617-29.

13. Dhawan V, Kazumata K, Robeson W, Belakhlef A, Margouleff C, Chaly T, et al. Quantitative brain PET. Comparison of 2D and 3D acquisitions on the GE Advance scanner. Clin Positron Imaging 1998;1:135-44.

14. Boecker H, Ceballos-Baumann A, Bartenstein P, Weindl A, Siebner HR, Fassbender T, et al. Sensory processing in Parkinson's and Huntington's disease: investigations with $3 \mathrm{D} \mathrm{H}_{2}{ }^{15} \mathrm{O}-\mathrm{PET}$. Brain 1999;122:1651-65.

15. Knesaurek K, Machac J, Krynycki B, Almeida O. Comparison of 2-dimensional and 3-dimensional 82- $\mathrm{Rb}$ myocardial perfusion PET imaging. J Nucl Med 2003;44:1350-6.

16. Schäfers KP, Spinks TJ, Camici PG, Bloomfield PM, Rhodes CG, Law MP, et al. Absolute quantification of myocardial blood flow with $\mathrm{H}_{2}{ }^{15} \mathrm{O}$ and 3-dimensional PET: an experimental validation. $\mathrm{J}$ Nucl Med 2002;43:1031-40.

17. Votaw JR, White M. Comparison of 2-dimensional and 3dimensional cardiac 82-Rb PET studies. J Nucl Med 2001; 42:701-6.

18. Lubberink M, Boellaard R, van der Weerdt AP, Visser FC, Lammertsma AA. Quantitative comparison of analytic and iterative reconstruction methods in 2- and 3-dimensional dynamic cardiac ${ }^{18}$ F-FDG PET. J Nucl Med 2004;45:2008-15.

19. Brogsitter C, Grüning T, Weise R, Wielepp P, Lindner O, Korfer R, et al. ${ }^{18} \mathrm{~F}-\mathrm{FDG}$ PET for detecting of myocardial viability. Validation of $3 \mathrm{D}$ data acquisition. J Nucl Med 2005;46:19-24.

20. Adam LE, Zaers J, Ostertag H, Trojan H, Belleman ME, Brix G. Performance evaluation of the whole-body PET scanner ECAT EXACT HR+ following the IEC standard. IEEE Trans Nucl Sci 1997;44:1172-9.

21. Brix G, Zaers J, Adam LE, Bellemann ME, Ostertag H, Trojan H, et al. Performance evaluation of a whole-body PET scanner using the NEMA protocol. J Nucl Med 1997;38:1614-23. 
22. Defrise M, Kinahan PE, Townsend DW, Sibomana MC, Newport DF. Exact and approximate rebinning algorithms for 3-D PET data. IEEE Trans Med Imaging 1997;16:145-58.

23. Watson CC. New, faster, image-based scatter correction for $3 \mathrm{D}$ PET. IEEE Trans Nucl Sci 2000;1587-94.

24. Bergström M, Eriksson L, Bohm C, Blomqvist G, Litton J. Correction for scattered radiation in a ring detector positron camera by integral transformation of the projections. J Comput Assist Tomogr 1983;7:42-50.

25. Van der Weerdt AP, Klein LJ, Boellaard R, Visser CA, Visser FC, Lammertsma AA. Image-derived input functions for determination of MRGlu in cardiac F-18-FDG PET scans. J Nucl Med 2001;42:1622-9.

26. Patlak CS, Blasberg RG, Fenstermacher JD. Graphical evaluation of blood-to-brain transfer constants from multiple-time uptake data. J Cereb Blood Flow Metab 1983;3:1-7.

27. Bland JM, Altman DG. Statistical methods for assessing agreement between two methods of clinical measurement. Lancet 1986;1(8476):307-10.
28. Paans AMJ, Boerdijk SMM, Willemsen ATM, Pruim J. Source of impaired image quality in 3D whole-body FDG PET scanning. Eur J Nucl Med Mol Imaging 2004;31:1207.

29. Visvikis D, Griffiths D, Costa DC, Bomanji J, Ell PJ. Clinical evaluation of $2 \mathrm{D}$ versus $3 \mathrm{D}$ whole body PET image quality using a dedicated BGO PET scanner. Eur J Nucl Med Mol Imaging 2005;32:1050-6.

30. Halpern BS, Dahlbom M, Auerbach MA, Schiepers C, Fueger BJ, Weber WA, et al. Optimizing imaging protocols for overweight and obese patients: a lutetium orthosilicate PET/CT study. J Nucl Med 2005;46:603-7.

31. Lodge MA, Badawi RD, Gilbert R, Dibos PE, Line BR Comparison of 2-dimensional and 3-dimensional acquisition for ${ }^{18}$ F-FDG PET oncology studies performed on an LSO-based scanner. J Nucl Med 2006;47:23-31.

32. Mawlawi O, Podoloff DA, Kohlmyer S, Williams JJ, Stearns CW, Culp RF, et al. Performance characteristics of a newly developed PET/CT scanner using NEMA standards in 2D and 3D modes. J Nucl Med 2004;45(10):1734-42. 